\title{
A 26-Year-Old Male with a 14-Year History of Left Intermittent Testicular Torsion Treated with Self-Manual Reduction
}

\author{
Jeremy M. West Andrew J. Goates James A. Brown \\ Department of Urology, University of lowa Hospitals and Clinics, lowa City, IA, USA
}

\section{Key Words}

Intermittent testicular torsion - Orchidopexy •

Acute scrotum

\begin{abstract}
Intermittent testicular torsion presents with recurrent episodes of severe unilateral testicular pain with resolution of symptoms between episodes. We present a case of a 26-year-old man with a 14-year history of intermittent testicular pain presenting to the urology clinic for elective surgical evaluation. He reported monthly symptoms for many years and had learned to manually reduce the torsion with each episode. Diagnosis of intermittent testicular torsion was made based on patient history and treatment with bilateral orchiopexy resulted in complete symptom resolution and without any loss of testicular function.
\end{abstract}

Copyright $\odot 2017$ S. Karger AG, Basel

\section{Introduction}

Testicular torsion is among the most common causes of acute testicular pain and prompt diagnosis is critical in order to preserve testicular function. Acute testicular torsion is often reported in pediatric and adolescent populations and less frequently in adults $[1,2]$. Diagnosis is typically made based on clinical characteristics of a high-riding, swollen, exquisitely tender testis with the cremasteric reflex often absent and may also be aided

\section{KARGER}

Fax +4161306 1234

E-Mail karger@karger.com

www.karger.com
(C) 2017 S. Karger AG, Basel

Accessible online at: www.karger.com/cur by the use of Doppler ultrasound. Testicular loss and infertility secondary to blood-testis disruption are the most severe complications highlighting the urgency of need for correction. Attempt at manual reduction of torsion is recommended as a temporizing measure, but surgical intervention for definitive treatment is needed as soon as possible, generally in less than 12 hours from symptom onset [3].

Much less commonly, patients may present with a history of recurrent episodes of testicular pain with spontaneous resolution and meet criteria for a scheduled, elective orchiopexy. This is frequently termed intermittent testicular torsion (ITT) and is an important entity to recognize as each episode of torsion puts the patient at risk of needing emergent surgery. To our knowledge, this is the first case report which a patient manually reduced his testicular torsion at onset of symptoms for over a decade without surgical intervention or loss of testicular viability/function.

\section{Case Report}

A 26-year-old male initially presented to his primary care provider at age 12 following an episode severe, sudden onset unilateral testicular pain associated with nausea which resolved spontaneously after approximately 2 hours. He was diagnosed with possible resolved testicular torsion. Referral to a urologic specialist was not given at that time given the resolution of his pain. Approximately every month, the patient developed similar symptoms but did not seek medical care; citing embarrassment as 
the key reason. Instead he proceeded with manual reduction of the affected side within the scrotum and was able to achieve prompt resolution of pain.

For the next 14 years the patient reported having testicular pain on average of 1-2 $\times$ per month and with each episode the patient was able to identify the directionality of rotation and manually reduce the torsion. He reported that episodes generally lasted for 15 minutes to 1 hour, occurred at anytime including at night, with no identifiable initiating event. During this time the patient fathered 3 children without any fertility concerns. At the age of 26 , while attending medical school, the rigors of training and frequent demand to interrupt study to reduce the torsion caused him to seek medical attention at an outpatient urology clinic. Physical examination revealed bilateral descended testes with no masses and a slightly smaller left testicle on palpation. It was suspected he had a case of ITT. The discussion was held to proceed with watchful waiting with ultrasound at time of symptoms versus bilateral orchidopexy. The patient elected for bilateral orchidopexy.

During the operation, both the left and right testicle were inspected. The right side revealed a small varicocele but otherwise no further abnormalities were seen bilaterally. Bilateral orchidopexy was performed using 3-0 Prolene to tack the tunica albuginea down to the dartos in the medial, lateral and inferior quadrants taking care to not twist the cord. At 6 months, the patient reported complete resolution of his symptoms, no further need for manual reduction, and complete returned to regular activities and exercise.

\section{Discussion}

Testicular torsion is an important consideration in all young males presenting with acute testicular pain. True torsion, is a surgical emergency and requires immediate intervention. ITT is generally characterized by pain resolution and asymptomatic periods between episodes [4]. Many episodes may occur prior to presentation for surgery. In 1 report the mean number of painful episodes before surgery was 4.3 [5]. In the case presented here, the patient experienced more than 100 episodes over a 14-year period, which is significantly more than has been reported previously. To our knowledge, this is the first case of such prolonged manual reduction of testicular torsion. Furthermore, the patient had no viability/ functionality issues with the testicle including fertility or sexual dysfunction.

While this is a rare presentation in modern healthcare, this case is similar to the initial description of ITT in 1895 by Van der Poel. He reported a 25-year-old student doctor who had ITT and learned to unwind his own spermatic cord to relieve his pain [6]. This patient also ultimately underwent surgical correction.

A recent study assessed the optimal management of patients with ITT. In particular, they looked at surgical intervention versus conservative management. Of the 63

A Unique Case of Longstanding

Intermittent Testicular Torsion patients included in the study, those undergoing scrotal orchidopexy were found to have significantly better pain resolution and symptom improvement [7].

The success of testicular salvage is multifactorial and depends on age of onset, duration of torsion and degree of torsion. Most studies to date have evaluated fertility status after acute torsion rather than elective surgery for intermittent torsion. A review article by Mellick et al. [8] outlines an extensive review of many series reports of the viability of testes after torsion with degree of torsion and duration of symptoms being the 2 most common factors associated with increased risk of testicular loss. Another recent study of 144 cases of torsion showed that the median duration of symptoms in the salvaged testes group was 6 hours and the median duration in the orchiectomy group was 46 hours [9]. This further adds to the vast body of studies indicating that although it is still possible and worth pursuing salvage of the testicle later, ideally corrective surgery should be done prior to 6 hours after pain onset.

When fertility is used as the ultimate endpoint rather than salvage of the testicle, prior studies suggest that the development and compensatory effort in the contralateral testicle is often normal and can often result in normal fertility [10]. Previous reports suggest that duration of symptoms lasting $>6$ hours is more likely to result in infertility. It is remarkable that this patient's fertility remained intact given the number of episodes this patient experienced prior to surgical correction. We suspect this patient's fertility remained intact due to prompt reductions of torsion with a single episode never lasting longer than approximately 1 hour.

This case highlights the importance of primary care providers to recognize ITT as a possible cause of testis pain and have a low threshold for prompt urology referral when suspected. As in this case, adolescents are understandably reluctant to seek care for testicular complaints and when they do seek care, it may be the only opportunity to intervene and prevent further complications. With an initial referral, the patient may have undergone orchidopexy much sooner reducing risk of emergent torsion and testicular loss as well as significantly improving his quality of life.

\section{Conclusion}

Testicular torsion is an important and common cause of acute testicular pain and can occur intermittently even for many years in some patients. Prompt diagnosis is crit- 
ical in order initiate surgical therapy to increase likelihood of preserve testicular function. This case highlights several important principles for the management of ITT including an atypical, delayed presentation, the importance of early urologic referral even with resolution of symptoms, and discussion of infertility risk with torsion.

\section{References}

1 Blumberg JM, White B, Khati NJ, Andrawis R: Intermittent testicular torsion in a 58-yearold man. J Urol 2004;172:1886.

2 Tolia BM, Newman HR: Testicular torsion in adults: plea for its consideration early in clinical evaluation of acute scrotum. Urology 1977;10:150-151.

3 Dunne PJ, O'Loughlin BS: Testicular torsion: time is the enemy. Aust N Z J Surg 2000;70:441-442.

4 Stillwell TJ, Kramer SA: Intermittent testicular torsion. Pediatrics 1986;77:908-911.
5 Eaton SH, Cendron MA, Estrada CR, Bauer SB, Borer JG, Cilento BG, Diamond DA, Retik AB, Peters CA: Intermittent testicular torsion: diagnostic features and management outcomes. J Urol 2005;174:1532-1535.

6 Van Der Poel J: Strangulation of the testis and epididymis from torsion of the spermatic cord. Med Rec NY 1895;37:737-742.

7 Al-Kandari AM, Kehinde EO, Khudair S, Ibrahim H, ElSheemy MS, Shokeir AA: Intermittent testicular torsion in adults: an overlooked clinical condition. Med Princ Pract 2017;26:30-34.
8 Mellick LB: Torsion of the testicle: it is time to stop tossing the dice. Pediatr Emerg Care 2012;28:80-86.

9 Pogorelic Z, Mustapic K, Jukic M, Todori J, Mrkli I, Mešštrovi J, Juri I, Furlan D: Management of acute scrotum in children: a 25 year single center experience on 558 pediatric patients. Can J Urol 2016;23:8594-8601.

10 Puri P, Barton D, O’Donnell B: Prepubertal testicular torsion: subsequent fertility. J Pediatr Surg 1985;20:598-601. 